Nota

\title{
TRICOMAS FOLIARES EM TOMATEIRO COM TEORES CONTRASTANTES DO ALELOQUÍMICO 2-TRIDECANONA
}

\author{
Carlos Alberto Aragão ${ }^{1,3 *}$; Bárbara França Dantas ; Flávio Rodrigo Gandolfi Benites ${ }^{2,4}$ \\ ${ }^{1}$ Depto. de Agricultura e Melhoramento Vegetal - FCA/UNESP, C.P. 237 - CEP: 18603-970 - Botucatu, SP. \\ ${ }^{2}$ Graduando em Agronomia-UFLA, C.P. 37 - CEP: 37200-000 - Lavras, MG. \\ ${ }^{3}$ Bolsista CAPES. \\ ${ }^{4}$ Bolsista CNPq/PIBIC. \\ *Autor correspondente <caaragao@fca.unesp.br>
}

\begin{abstract}
RESUMO: Duas espécies de tomateiro, Lycopersicon esculentum Mill. (Linhagem TOM 556- padrão com baixo teor da metil cetona 2-tridecanona (2-TD) e Lycopersicon hirsutum Dunal var. glabratum Mill. (Linhagem PI 134417- padrão com elevado teor de 2-TD), foram analisadas em conjunto para identificação e quantificação de tricomas foliares. A parte experimental constou da identificação dos tricomas, baseado na presença ou ausência da cabeça secretora na extremidade apical dos tricomas, arranjo e número de células da cabeça, comprimento dos tricomas, e da quantificação do número de tricomas glandulares e não glandulares nos folíolos. O delineamento utilizado para o número de tricomas foi inteiramente casualizado e as médias comparadas pelo teste de Tukey, a 5\%. A identificação e quantificação para as espécies foram: a) TOM 556: Tricomas não glandulares do tipo II + III + V $\left(2590 \mathrm{~cm}^{-2}\right.$ de área foliolar); glandular do tipo VI (16 cm $\mathrm{cm}^{-2}$; glandular do tipo VII (138 $\left.\mathrm{cm}^{-2}\right)$; b) PI 134417: não glandulares do tipo II + III + V (140 $\mathrm{cm}^{-2}$ de área foliolar); glandular do tipo I + IV $\left(843 \mathrm{~cm}^{-2}\right)$; glandular do tipo VI $\left(83 \mathrm{~cm}^{-2}\right)$; glandular do tipo VII $\left(11 \mathrm{~cm}^{-2}\right)$. As maiores concentrações da 2-TD nos folíolos, estão associadas às maiores densidades de tricomas glandulares presentes em ambas espécies.
\end{abstract}

Palavras-chave: Lycopersicon esculentum, tomate, tricomas, aleloquímico, 2-tridecanona

\section{FOLIAR TRICHOME IN TOMATO WITH CONTRASTING LEVELS OF ALELOCHEMICAL 2-TRIDECANONE}

\begin{abstract}
Two tomato species, Lycopersicon esculentum Mill. (Line TOM 556), which contained low levels of the methyl ketone 2-tridecanone (2-TD) and Lycopersicon hirsutum Dunal var. glabratum Mill. (Line PI 134417), with high levels of 2-TD, were evaluated for identification and quantification of foliar trichomes. The experiment was carried out to identify the trichomes, based on their length and on the presence, form, cell number and cell arrangment of secretory heads, as well as to quantify glandular and non-glandular trichomes. The experimental design used for trichome number was totally randomized and the averages were compared by the Tukey test, $5 \%$. The identification and quantification of trichomes for the two species were: a) TOM 556: Types II + III + V non-glandular trichomes (2590 $\mathrm{cm}^{-2}$ of foliar area); Type VI glandular trichome $\left(16 \mathrm{~cm}^{2-}\right)$; Type VII glandular trichome $\left(138 \mathrm{~cm}^{-2}\right)$; b) PI 134417: Types II + III + V non-glandular trichomes $\left(140 \mathrm{~cm}^{-2}\right)$; Type I + IV glandular trichomes $\left(843 \mathrm{~cm}^{-2}\right)$; Type VI glandular trichome $\left(83 \mathrm{~cm}^{-2}\right)$; Type VII glandular trichome $\left(11 \mathrm{~cm}^{-2}\right)$. High concentrations of 2-TD were associated with higher densities of glandular trichomes in both species.

Key words: Lycopersicon esculentum, tomato, trichome, allelochemic, 2-tridecanone
\end{abstract}

\section{INTRODUÇÃO}

Tricomas foliares exercem um papel fundamental na defesa de plantas, principalmente em relação a insetos fitófagos. Em várias espécies há uma correlação negativa entre a densidade de tricomas e as respostas de alimentação, oviposição de insetos adultos e nutrição da larvas (Theobald et al., 1979). Os tricomas não glandulares podem atuar diretamente sobre os insetos, afetando sua oviposição, alimentação, locomoção, ou seu comportamento em relação ao abrigo, através de sua densidade e tamanho. Os tricomas glandulares podem, também, ser complementos na defesa química devido à secreção de terpenos, alcalóides, substâncias fenólicas e outras que podem ser repelentes olfatórias ou gustatórias.

De acordo com Lara (1991), em tomateiro os tricomas podem proporcionar proteção, limitando o acesso de insetos à superfície da planta, ou devido a sua densidade e comprimento ou através de produção de toxinas que atuam direta ou indiretamente sobre os insetos. Tricomas glandulares em espécies silvestres exsudam substâncias tóxicas, como a 2-tridecanona (2-TD), um líquido colante que pode prender os insetos à planta (Lara, 1991); além disso, a substância pode acumular-se no labium do inseto, impedindo a sucção da seiva (Gibson, 1971).

As espécies de Lycopersicon podem apresentar grandes variações nos tipos de tricomas entre si. Sendo 
eles, glandulares ou não glandulares, unicelulares ou multicelulares e com células da base diferenciadas ou não. Os glandulares são geralmente capitados. A cabeça, que é a região secretora, pode ser unicelular ou multicelular. As células da cabeça são revestidas pela cutícula (Cutter, 1986; Theobald et al., 1979). Luckwill (1943) descreveu, no gênero Lycopersicon sete tipos de tricomas: não glandulares (tipos II, III e V) e glandulares (I, IV,VI e VII). O tipo VI existente na espécie selvagem $L$. hirsutum apresenta cabeça multicelular, é o de maior importância na resistência a insetos. A densidade dos tricomas glandulares do tipo $\mathrm{VI}$ assume maior importância, para seleção indireta, em programas de melhoramento visando resistência a artrópodes. Essa característica pode ser influenciada por diversos fatores, como comprimento do dia e idade da planta. São observadas maiores densidades de tricomas tipo Vl em dias longos (Good Junior \& Snyder, 1988) e plantas jovens (Lin et al., 1987).

Acredita-se que na cabeça desses tricomas esteja contido o maior teor da toxina 2-tridecanona (2-TD) em plantas de L. hirsutum Dunal var. glabratum Mill. (Kennedy \& Sorenson, 1985), sendo encontrada também em pequenas quantidades em L. esculentum Mill. (Fery \& Kennedy, 1987). Williams et al. (1980) relataram que o aleloquímico 2-TD é o princípio ativo da resistência a insetos encontrado em $L$. hirsutum Dunal var. glabratum Mill. (PI 134417). Segundo Kennedy \& Sorenson (1985), a 2-TD está associada aos tricomas glandulares que revestem as folhas. Esta metil cetona é praticamente ausente no interior das folhas, estando concentrada mais na superfície das mesmas onde se encontram os tricomas glandulares.

Este estudo foi realizado com o objetivo de identificar e quantificar tricomas foliares em dois acessos de tomateiro das espécies Lycopersicon hirsutum Dunal var. glabratum Mill. e Lycopersicon esculentum Mill., contrastantes em teores de 2-tridecanona em seus folíolos.

\section{MATERIAL E MÉTODOS}

O experimento foi conduzido nos meses de setembro-outubro de 1997, no laboratório de Anatomia Vegetal, do Departamento de Biologia da Universidade Federal de Lavras- MG, foram avaliados os acessos PI 134417 (Lycopersicon hirsutum Dunal var. glabratum Mill., com alto teor de 2-tridecanona (2-TD) e TOM 556 ( $L$. esculentum Mill., com baixo teor de 2-TD).

As plantas usadas para este estudo foram cultivadas sob condições de estufa, em vasos de 15 litros com solo, onde as plantas receberam todos os tratos culturais empregados para a cultura na região. Em 10 plantas de cada acesso, aos 50 dias de idade, foram coletados 2 folíolos do terço médio de cada espécime, região em que estes encontravam-se completamente expandidos, os quais foram fixados em álcool etílico a 70\% (Jensen, 1962) por 72 horas para confecção de cortes paradérmicos.

Para cada corte paradérmico, fez-se a identificação, medição de comprimento e quantificação dos tricomas conforme indicado a seguir.
Os cortes paradérmicos foram realizados nas faces abaxial e adaxial dos folíolos, nas regiões apical, mediana e basal de cada face, com auxílio de uma lâmina de aço. Os mesmos foram corados com safranina $0,1 \%$ em água + glicerina, sendo montadas lâminas semipermanentes que foram observadas em microscópio OLYMPUS CBB, acoplado com uma câmara clara.

Os tricomas foram identificados de acordo com a presença ou ausência de cabeça em sua extremidade apical, número e arranjo das células da cabeça e comprimento dos tricomas e presença de células diferenciadas na base dos tricomas, de acordo com Luckwill (1943) e Theobald et al. (1979).

Efetuou-se a medição do comprimento dos tricomas, os quais foram projetados num campo de dimensão conhecida com auxílio de uma câmara clara. $\mathrm{Na}$ projeção formada, desenhou-se uma linha por toda a extensão do tricoma projetado, o que representa o seu comprimento. Posteriormente a linha marcada foi medida com auxílio de um curvímetro. O comprimento dos tricomas foram expressos em micrômetros.

A quantificação foi realizada também com auxílio de projeção em campo de dimensão conhecida, onde os tricomas projetados foram contados, técnica esta adaptada de Labouriau et al. (1961), que a utilizaram para contagem de estômatos.

Para os genótipos avaliados TOM 556 e PI 134417 adotou-se o mesmo critério para identificação, quantificação e medição do comprimento dos tricomas. O número de

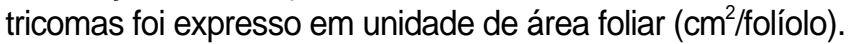

$O$ delineamento utilizado foi inteiramente casualizado e as médias comparadas pelo teste de Tukey, a $5 \%$.

\section{RESULTADOS E DISCUSSÃO}

Todos os tricomas glandulares identificados são simples (não ramificados), de pedúnculo uniseriado e multicelulares (Figura1), sendo reconhecidos quatro tipos:

A- Tricoma glandular do tipo I: Apresenta o maior comprimento em comparação aos demais tricomas glandulares $(700-2000 \mu \mathrm{m})$. Tem células da base diferenciadas e presença de cabeça unicelular em sua extremidade apical (Figura 1-A);

B- Tricoma glandular do tipo IV: Assemelha-se ao tipo I, tendo como diferenças básicas o seu comprimento (200-400 $\mu \mathrm{m})$ e células basais semelhantes às do pedúnculo. (Figura 1-B);

C- Tricoma glandular do tipo VI: Tem comprimento que varia entre $200-400 \mu \mathrm{m}$ com pedúnculo que se afila da base para o ápice, encimado por uma cabeça multicelular e tetralobular. Tem células da base diferenciadas (Figura 1-C). É considerado o de maior importância para resistência a artrópodes, por apresentar as maiores concentrações da 2-TD (Williams et al., 1980);

D- Tricoma glandular do tipo VII: Apresenta cabeça multicelular tetralobular, bem mais evidenciada que a do 
tipo VI; seu pedúnculo mantém-se com diâmetro uniforme desde a base até a extremidade apical. É o menor tricoma, com comprimento de 50 a $100 \mu \mathrm{m}$ (Figura 1-D);

Os tricomas não glandulares dos tipos II, III e V são bastante semelhantes entre si, diferindo basicamente em comprimento. Estes têm importância secundária, pois acredita-se que o aleloquímico natural 2-TD esteja contido nos tricomas glandulares capitados (Williams et al., 1980).

Os dados referentes à densidade de tricomas glandulares e não glandulares nos genótipos $L$. esculentum Mill. (TOM 556) e a espécie selvagem $L$. hirsutum Dunal var. glabratum Mill. (PI 134417) estão apresentados na TABELA 1.

Verifica-se através das quantificações realizadas nos genótipos avaliados, uma maior predominância de tricomas glandulares (tipos: I, IV e VI) para PI 134417, quando comparado a TOM 556. Os tricomas glandulares, principalmente os do tipo $\mathrm{VI}$ mostraram-se em maiores densidades em folíolos de PI 134417, em relação a TOM 556. Espécies silvestres, como é o caso do PI 134417, apresentam, segundo, Weston et al. (1989), maior densidade de tricomas glandulares em seus folíolos, o que confere a esses genótipos maior tolerância a Helicoverpa zea Bod. (Dimock et al., 1982), Manduca sexta L. (Schwartz \& Snyder, 1983), Leptinotarsa decemlineata Say. (Kennedy \& Sorenson, 1985), Keiferia lycopersicella Wals. (Lin et al., 1987), Scrobipalpuloides absoluta Meyrick. (Giustolin, 1991), Spodoptera exigua (Lin et al., 1987; Eigenbrone \& Trumble,
1993) e Phithorimaea operculella Zell. (Ventura \& Vendramim, 1995), quando relacionados a espécies cultivadas. Williams et al. (1980) e Dimock et al. (1982) constataram a ocorrência da metil cetona na superfície dos folíolos do acesso silvestre de tomateiro PI 134417. Aragão (1998) estabeleceu uma correlação positiva significativa entre a concentração de 2tridecanona e a densidade de tricomas glandulares do tipo $\mathrm{VI}$, relacionados à resistência ao ácaro rajado (Tetranychus urticae Koch.).

Barbosa (1994) e Labory (1996) verificaram a resistência de $\mathrm{PI} 134417$ à traça-do-tomateiro (Tuta absoluta Meyrick.) e ao ácaro rajado (Tetranychus urticae Koch.), devido à existência de maiores densidades dos tricomas do tipo VI nos folíolos, confirmando trabalhos realizados por Kennedy \& Yamamoto (1979).

Apesar de evidências disponíveis sugerirem que tricomas do tipo VI de folíolos de L. hirsutum Dunal var. glabratum Mill. ser a principal fonte de 2-TD (Barbosa, 1994; Dimock \& Kennedy (1983), Good Junior \& Snyder (1988) reportam que os tricomas glandulares do tipo IV também estão envolvidos na produção do inseticida natural, 2-TD.

Devido à facilidade, baixo custo e alta correlação com resistência a várias pragas, a avaliação do número de tricomas glandulares em folíolos de tomateiro pode ser usada como técnica indireta de seleção para resistência a artrópodes. Esta avaliação, através de cortes paradérmicos, pode ser usada na seleção de plantas com alta densidade de tricomas glandulares, em populações
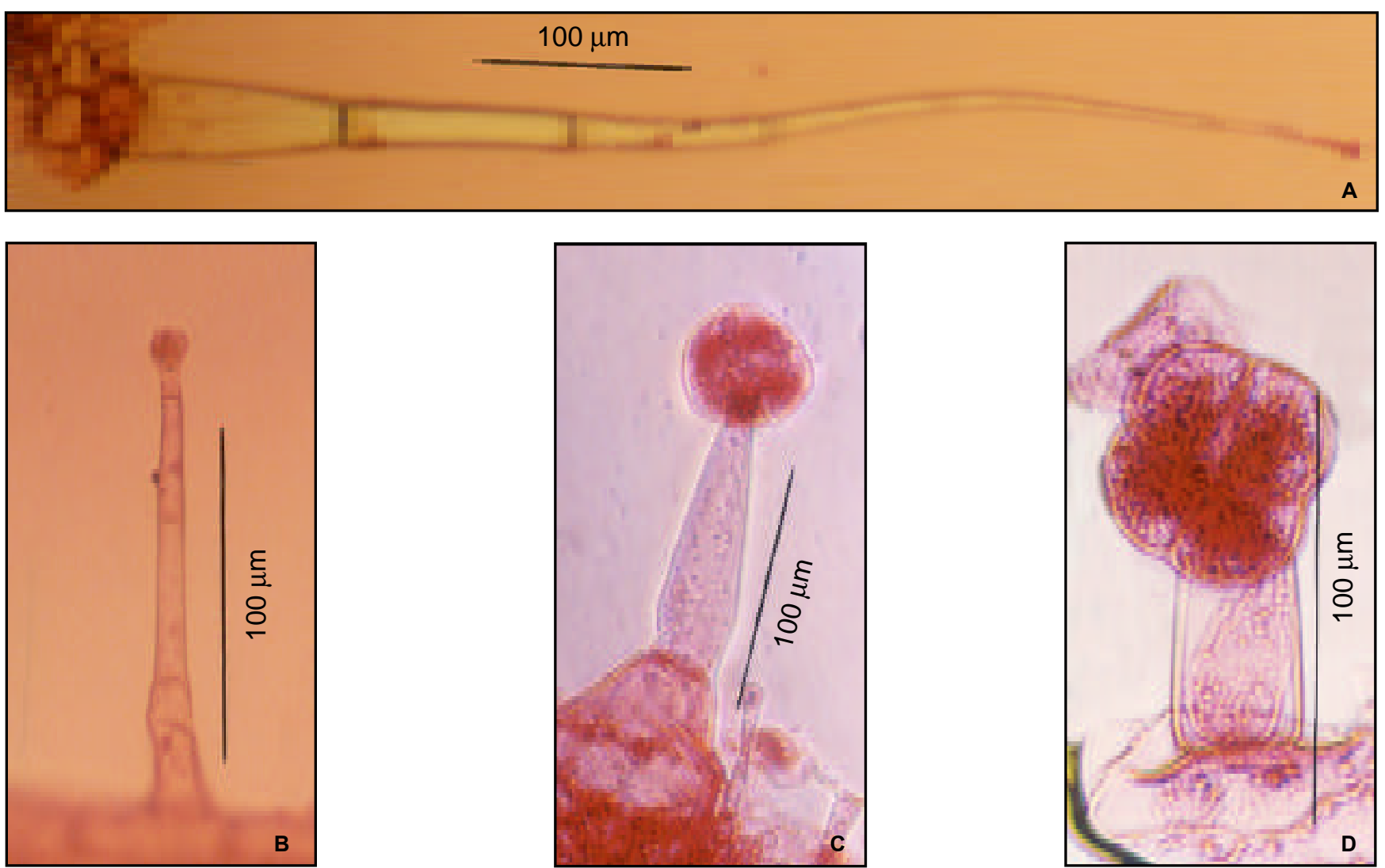

Figura 1 - Tricomas glandulares em folíolos de duas espécies de tomateiro, Lycopersicon esculentum Mill. (TOM 556) e Lycopersicon hirsutum Dunal var. glabratum Mill. (PI 134417). Tricomas tipo I (A), tipo IV (B), tipo VI (C) e tipo VII (D). 
TABELA 1 - Densidade de tricomas glandulares e não glandulares nos genótipos de tomateiro TOM 556 e PI 134417. UFLA, Lavras MG, 1997

\begin{tabular}{lcccc}
\hline Genótipos & \multicolumn{5}{c}{$\mathrm{N}^{\circ}$ de tricomas por $\mathrm{cm}^{2}$ de área foliolar } \\
\hline nOa glandulares Tipo II + III + V & glandulares Tipo I + IV & glandulares Tipo VI & glandulares Tipo VII \\
\hline PI 134417 & $2.590 \mathrm{a}$ & 0 & $16 \mathrm{~b}$ & $138 \mathrm{a}$ \\
\hline
\end{tabular}

Médias seguidas de mesma letra não diferem pelo teste Tukey, a 5\%.

segregantes, oriundas de cruzamentos interespecíficos de Lycopersicon esculentum Mill, com a espécie silvestre Lycopersicon hirsutum Dunal var. glabratum Mill. em programas de melhoramento visando resistência a pragas.

\section{CONCLUSÕES}

- A espécie silvestre $L$. hirsutum Dunal var. glabratum Mill. (PI 134417) - padrão para alto teor de 2-TD apresentou maior densidade de tricomas glandulares em seus folíolos, quando comparada à espécie cultivada $L$. esculentum Mill. (TOM 556) - padrão para baixo teor de 2-TD.

- A seleção para maiores densidades de tricomas glandulares em plantas de uma população segregante de L. esculentum Mill x L. hirsutum Dunal var. glabratum pode ser válida como técnica indireta de seleção para resistência a artrópodes em Lycopersicon, de grande importância em programas de melhoramento.

\section{REFERÊNCIAS BIBLIOGRÁFICAS}

ARAGÃO, C.A. Tricomas foliares associados à resistência ao ácaro rajado Tetranychus urticae Koch. em linhagens de tomateiro com alto teor de 2-trideacnona nos folíolos. Lavras, 1998. 71p. Dissertação (Mestrado) - Universidade Federal de Lavras.

BARBOSA, L.V. Controle genético e mecanismos de resistência em Lycopersicon spp à traça do tomateiro [Scrobipalpuloides absoluta (Meyrick, 1917) (Lep. Gelechiidae)]. Lavras, 1994. 71p. Dissertação (Mestrado) - Universidade Federal de Lavras.

CUTTER, E.G. Anatomia vegetal: Parte I. Células e tecidos. 2.ed. São Paulo: Roca, 1986. 303p.

DIMOCK, M.B.; KENNEDY, G.G. The role of glandular trichomes in the resistance of Lycopersicon hirsutum f. glabratum to Heliothis zea. Entomologia Experimentalis et Applicata, v.33, p.263-268, 1983.

DIMOCK, M.B.; KENNEDY, G.G.; WILLAMS, W.G. Toxicity of analogs of 2-tridecanone, a naturally occurring toxicant from a wild tomato. Journal of Chemical Ecology, v.8, p.837-842, 1982.

EIGENBRONE, S.D.; TRUMBLE, J.T. Antibiosis to beet armyworm (Spodoptera exigua) in Lycopersicon accesions. HortScience, v.28, p.932-934, 1993.

FERY, R.L.; KENNEDY, G.G. Genetic analysis of 2-tridecanone concentration, leaf trichome characteristics, and tobacco hornworm resistance in tomato. Journal of the American Society for Horticultural Science, v.5, p.886-891, 1987.

GIBSON, R.W. Glandular hairs provinding resistance to aphids in certain wild potato especies. Annals of Applied Biology, v.68, p.113-119, 1971.

GIUSTOLIN, T.A. Efeito dos aleloquímicos 2-tridecanona e 2-undecanona, presentes em Lycopersicon spp., sobre a biologia da traçar do tomateiro Scrobipalpuloides absoluta (Meyrick, 1917). Piracicaba, 1991. 155p. Dissertação (Mestrado) - Escola Superior de Agricultura "Luiz de Queiroz", Universidade de São Paulo.
GOOD JUNIOR, D.E.; SNYDER, J.C. Seasonal variation of leaves and mite resistance of Lycopersicon interespecific hybrids. HortScience, v.23, p.891- 894, 1988.

JENSEN, W.A. Botanical histochemistry; principles and pratice. San Francisco: W. H. Freeman, 1962. 408p.

KENNEDY, G.G.; SORENSON, C.F. Role of glandular trichomes in the resistance of Lycopersicon hirsutum f. glabratum to Colorado potato beetle (Coleoptera: Chrysomelidae). Journal of Economic Entomology, v.78, p.547-555, 1985.

KENNEDY, G.G.; YAMAMOTO, R.T.A. Toxic factor causing resistance in a wild tomato to the tobacco hornworm and some other insects. Entomologia Experimentalis et Applicata, v.26, p.121-126, 1979.

LABORY, C.R.G. Repetibilidade e herdabilidade do teor de 2tridecanona em folíolos de tomateiro e sua relação com a resistência a traça-do-tomateiro scrobipalpuloides absoluta (MEYRICK 1917). Lavras, 1996. 49p. Dissertação (Mestrado) - Universidade Federal de Lavras.

LABOURIAU, L.G.; OLIVEIRA, J.G.; SALGADO-LABOURIAU, M.L. Transpiração de Schizolobium parahyba (VELL.) Toledo: I. Comportamento na estação chuvosa, nas condições de Caeté, Minas Gerais, Brasil. Anais da Academia Brasileira de Ciências, v.33, p.237-257, 1961.

LARA, F.M. Princípios de resistência de plantas aos insetos. 2.ed. São Paulo: Ícone, 1991. 336p.

LIN, S.Y.H. TRUMBLE, J.T.; KUMAMOTO, J. Activity of volatile compounds in glandular trichomes of Lycopersicon species against two insect herbivores. Journal of Chemical Ecology, v.13, p.837-850, 1987.

LUCKWILL, L.C. The genus Lycopersicon: an historical, biological, and taxonomic survey of the wild and cultivated tomatoes. Aberdeen: University Press, 1943. 44p.

SCHWARTZ, R. F.; SNYDER, J. C. Characterization of resistance to tobacco hornworn in Lycopersicon leaflets. HortScience, v.18, p.170-174, 1983.

THEOBALD, W. L.; KRAHULIK, J. L.; ROLLINS, R. C. Trichome description and classification. In: METCALFE, C.R.; CHALK, L. (Ed.) Anatomy of dicotyledons. 2ed. Oxford: Oxford Science, 1979. v.1, cap.5, p.41-53.

VENTURA, M.U.; VENDRAMIM, J.D. Toxicidade para lagartas de Phthorimae operculella (Zell.) dos aleloquímicos 2tridecanona e 2-undecanona presentes em tomateiro (Lycopersicon spp.). Scientia Agricola, v.52, p.458-461, 1995.

WESTON, P.A.; JOHNSON, D.A.; BURTON, H.T.; SNYDER, J.C. Trichome secretion composition, trichome densities, and spider mite resistance of tem accessions of Lycopersicon hirsutum. Journal of the American Society for Horticultural Science, v.114, p.492-498, 1989.

WILLIAMS, W.G.; KENNEDY, G.G.; YAMAMOTO, E.T.; THACKER, J.D.; BORDNER, J. 2-tridecanone: a naturally occurring insetcticide from the wild tomato Lycopersicon hirsutum f. glabratum. Science, v.207, p.888-889, 1980.

Recebido em 23.11.99 